Document downloaded from:

http://hdl.handle.net/10251/94988

This paper must be cited as:

Gerardy, I.; Ródenas Diago, J.; Van Dycke, M.; Gallardo Bermell, S.; Ceccolini, E. (2010). Dosimetric characterization of a brachytherapy applicator using MCNP5 modelisation and inphantom measurements. Applied Radiation and Isotopes. 68(4):735-737. doi:10.1016/j.apradiso.2009.10.018

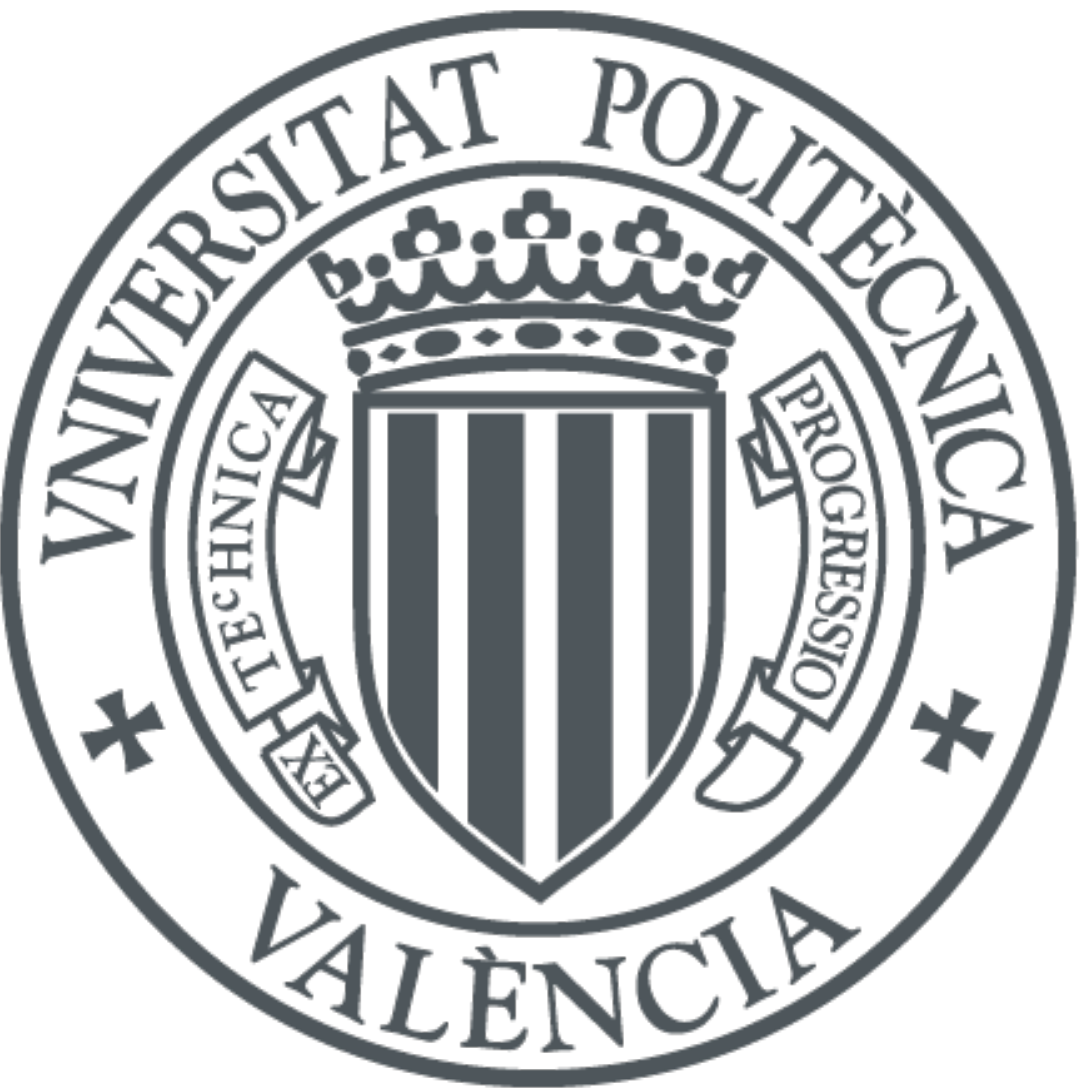

The final publication is available at

https://doi.org/10.1016/j.apradiso.2009.10.018

Copyright Elsevier

Additional Information 


\title{
Dosimetric characterization of a brachytherapy applicator using MCNP5 modelisation and in-phantom measurements.
}

\author{
José Ródenas ${ }^{1}$, Isabelle Gerardy ${ }^{2}$, Michel van Dycke ${ }^{3}$, Sergio Gallardo ${ }^{1 *}$, Elisa \\ Ceccolini $^{4}$
}

${ }^{1}$ Departamento de Ingeniería Química y Nuclear, Universidad Politécnica de Valencia, Apartado 22012, E-46071 Valencia (Spain)

${ }^{2}$ Institut Supérieur Industriel de Bruxelles, 150, Rue Royale, B-1000 Brussels (Belgium)

${ }^{3}$ Clinique Saint Jean, Bld du Jardin Botanique, B-1000 Brussels (Belgium)

${ }^{4}$ Facolta di Ingegneria, Alma Mater Studiorum Universita di Bologna (Italy)

\begin{abstract}
Brachytherapy is a radiotherapy treatment where encapsulated radioactive sources are introduced permanently (interstitial seeds) or temporally (interstitial or intracavitary devices) within a patient. When high dose rate sources (HDR) are used in intracavitary treatments, high doses can be locally imparted within the patient body. Monte Carlo simulation codes are powerful tools to simulate sources and devices to help physicists in treatment planning as well as to evaluate the dose received by the patient. In multiple types of gynaecological cancer, intracavitary brachytherapy can be used combined with other therapy treatment to give an additional local dose to the tumour. In these cases, different types of applicators can be used in order to increase the dose imparted to the tumour while the effect on healthy tissues is restricted. Due to the proximity of some more radiosensitive organs (i.e. rectum), the dose distribution into the tissue near the applicator has to be known as precisely as possible in order to avoid damage to these organs. The aim of this work is to model both applicator and source in order to evaluate the dose at a reference point as well as the effect of the materials constituting the applicators on the near field dose. The MCNP5 code based on the Monte Carlo method has been used for the simulation. A gynaecological applicator, consisting of a metallic intra-uterine tube with a plastic vaginal applicator, and a Microselectron HDR Ir-192 source have been simulated to evaluate the dose distribution. A solid phantom (PMMA) has been designed to perform measurements around the applicator with radiochromic films (type Gafchromic EBT). The isodose curves obtained are compared with curves calculated with the F4MESH tally of MCNP5.
\end{abstract}

KEYWORDS: brachytherapy, Monte Carlo method, MCNP5, dosimetry, radiochromic films, pinpoint chamber

\section{Introduction}

Brachytherapy consists in using an encapsulated source at short distance from the target volume for irradiation of malignant tumour or non-malignant lesions. It can be used alone or in combination with an external radiotherapy treatment. Compared to external beam treatment, the physical advantages of brachytherapy result in a better localization of the dose into the tumour and better protection of healthy tissues. In the case of intracavitary afterloaded high dose rate treatment (HDR), a source of high activity is temporary introduced into the patient body. Depending on the tumour localization, different applicators devices can be used to achieve a right positioning of the source. Due to the high dose rate, dose distribution has to be known with a good precision to perform the treatment planning. It has to be evaluated in the near field to ensure that the tumour receive the prescribed dose but also at larger distances to evaluate the dose imparted to organs with higher risk like bladder or rectum for a gynaecological treatment. In the AAPM TG 56 [1], quality assurance advices are given to ensure the good quality of the treatment and the security of the patient. These requirements are focused on the control of the irradiation time, the source localisation and the dose distribution around the applicator device. To meet the quality requirements for dose evaluation, both measurements and Monte Carlo

\footnotetext{
* Presenting author, E-mail: sergalbe@iqn.upv.es
} 
calculations can be used. In the present work, the case of HDR treatment and gynaecological applicator (consisting of an intra-uterine tube and vaginal cylinders) has been investigated

\section{Instrumentation and method}

\subsection{Modelling of the source and applicator}

The source considered in this work is an HDR Ir-192 Microselectron of Nucletron. A model of the source including its capsule (316L stainless steel) and guiding cable (304 stainless steel) has been developed. The energy spectrum was obtained from the JANIS database [2]. A layout of the source model can be seen in figure 1 .

Figure 1: source model

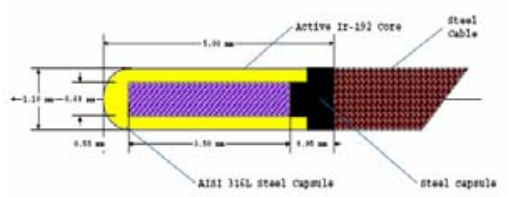

In the case of a gynaecological treatment, an applicator is used for both the right positioning of the HDR source and the protection of healthy tissues. The applicator used in this work consists of two parts: an intra-uterine tube and a vaginal applicator. The intra-uterine part consists of a metallic tube (AISI 304, density of $7.92 \mathrm{~g} / \mathrm{cm}^{3}$ ) with a $15^{\circ}$ inclination of the end side. The vaginal applicator is formed by two plastic cylinders (PPSU, density of $1.29 \mathrm{~g} / \mathrm{cm}^{3}$ ) of $2 \mathrm{~cm}$ diameter and $2.5 \mathrm{~cm}$ length. The model developed for MCNP5 calculation is shown on figure 2. The figure 3 represents an axial view of the source into the applicator tube.

Figure 2: applicator model

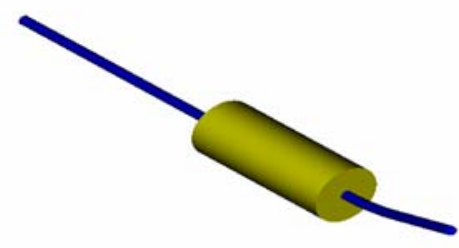

Figure 3: source into applicator

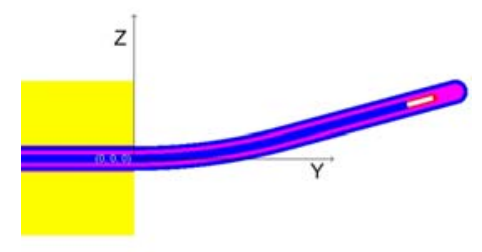

\subsection{MCNP5 Calculation}

The simulations have been performed using the MCNP5 code [3] based on the Monte Carlo method. Two types of calculation have been done for the dosimetric study of the irradiation device (source and applicator). For dose evaluations, the *F8 tally (photon and electron), which gives energy value deposit in a cell (in $\mathrm{MeV}$ ) has been used. For obtaining isodoses around applicator device, a F4MESH calculation, using DE and DF cards to transform fluence (particle/ $\mathrm{cm}^{3}-\mathrm{s}$ ) into dose (Gy), has been performed. Values for these cards have been obtained using the energy mass absorption coefficient provided by NIST [4]. The ENDF/B-VI cross section library has been used. The voxel size for calculation depends on the size of the measuring device used in the comparison between calculation and measurement. Only photon calculation has been done. The cutoff has been fixed to $1 \mathrm{keV}$ for photons and $20 \mathrm{keV}$ for electrons. In each case, 500 millions of particles have been launched to improve statistics [3]. 


\subsection{Design of the PMMA phantom}

To allow the good positioning of both measuring device and gynaecological applicator, a PMMA phantom has been developed. It consists of 30 PMMA sheets (density $1.19 \mathrm{~g} / \mathrm{cm}^{3}$ ) of $15^{*} 15^{*} 0.5 \mathrm{~cm}$. The four central sheets have been manufactured in order to contain the applicator. Other sheets allow the positioning of different measurement device, like radiochromic films or a small ionization chamber. To allow comparison between in-phantom measurements and MCNP5 calculations, a model of the phantom has been developed. The layout can be seen on figure 4 .

Figure 4: MCNP5 model of the PMMA phantom

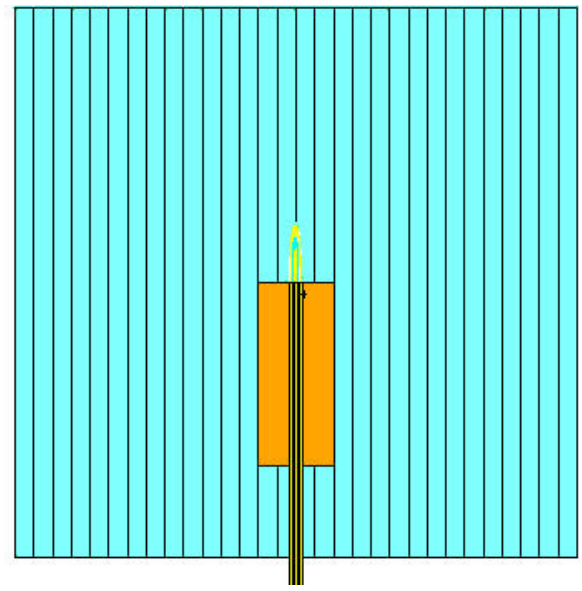

\subsection{Dose measurement devices}

Different devices have been used to perform the real measurements in the phantom.

\subsubsection{Radiochromic films}

Radiochromic films are self developing films consisting of a layer of Pentacosa-10,12-diynoic acid (37 $\mu \mathrm{m})$ between two layers of clear polyester (97 $\mu \mathrm{m}$ each). They are widely used for the evaluation of relative doses but also for absolute absorbed doses because of being tissue equivalent $\left(\mathrm{Z}_{\mathrm{eff}}=6.90\right)$ and showing a high spatial resolution. In this study, Gafchromic EBT( $)$ have been used to measure isodoses. Each film used has been scanned with a VIDAR VXR12 medical scanner to assess the uniformity of the reading (optical density, OD) before use. The dose calibration has been performed for 18 different doses ranging from 0 to 6.8 Gy delivered by a $6 \mathrm{MV}$ photon beam provided by a LINAC medical accelerator. The reading has been realized $12 \mathrm{~h}$ after irradiation using a VIDAR VXR 12 and the software RITT113 [6]. The spatial resolution of the reading is $85 \mu \mathrm{m}$ per pixel.

\subsubsection{Pinpoint ionization chamber}

In order to measure a point dose, a small open air ionization chamber CC04 of Wellhöfer (active volume of $0.04 \mathrm{~cm}^{3}, 3.6 \mathrm{~mm}$ length) has been used to evaluate the dose at some reference points, and at different distances from the source axis. The chamber has been calibrated for the energies of the ${ }^{60} \mathrm{Co}$ and a correction factor has been used for the ${ }^{192}$ Ir. It has been inserted into the PMMA phantom sheets.

\section{Discussion and results}

\subsection{Comparison of gafchromic measurements and MCNP5 calculation}

For the in-phantom measurement, the gafchromic film $(15 \mathrm{~cm} * 12,5 \mathrm{~cm})$ has been inserted between two PMMA sheets at $1 \mathrm{~cm}$ from the intra-uterine tube axis. The total dose is the sum of those delivered at four different positions of the source into the intra-uterine tube in order to minimize the dependence of 
the source position. After scanning with the VXR 12 and applying calibration (OD= function of dose), a dose matrix has been obtained. Isodose curves can be drawn using the RITT 113 software. These measured curves have been compared with those ones obtained using F4MESH tally of MCNP5. For this calculation, the voxel size has been fixed at $1 * 1 * 0.037 \mathrm{~mm}$ where $37 \mu \mathrm{m}$ corresponds to the thickness of the active layer in the Gafchromic film. Dose profiles can also be obtained. The figure 5 show the comparison of measured and calculated isodose curves. A maximum shift of $1 \mathrm{~mm}$ can be observed. The figure 6 shows a vertical profile of both measured and calculated doses taken in the center of the isodose curves. Both profiles are in good agreement. In general, the maximum difference between both dose matrices did not exceed $4 \%$.

Figure 5: Comparison between calculated and measured isodose curves

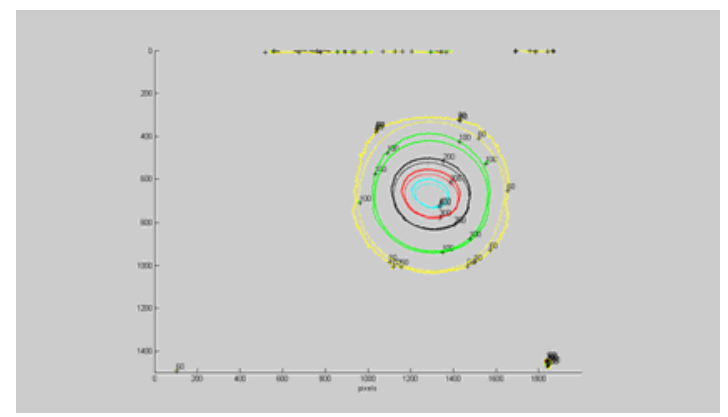

Figure 6 :Comparison between calculated and measured vertical dose profiles

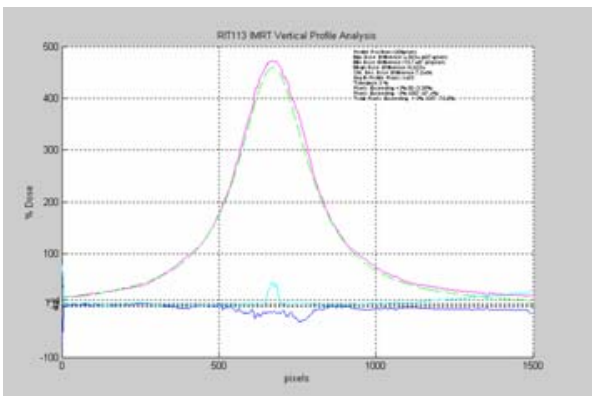

\subsection{Comparison between pinpoint dose measurement and MCNP5 calculation}

The Wellhöfer CC04 chamber has been inserted into the PMMA phantom at different distances of the intra-uterine tube axe (from $1.5 \mathrm{~cm}$ to $4 \mathrm{~cm}$, with a $0.5 \mathrm{~cm}$ step). Total dose measurement has been obtained with 4 positions of the source like explained in 3.1. Dose was calculated using calibration factor (provided by manufacturer, in water and for ${ }^{60} \mathrm{Co} \mathrm{N}_{\mathrm{D}, \mathrm{W}}=9.4810^{8} \mathrm{~Gy} / \mathrm{C}$ ) and correction factors for atmospheric pressure, temperature, isotope and materiel (PMMA). The absorbed dose was compared with two different MCNP5 calculations: a *F8 energy deposition tally in the active cavity of the chamber and an F4MESH (corrected with DE, DF cards calculated for the phantom material, see 3.1) calculation in a plane perpendicular to the intra-uterine tube axis that also contains the axis of the cylindrical CC04 chamber. Measurements and calculations have been made for distance ranging from $1.5 \mathrm{~cm}$ to $4 \mathrm{~cm}$ from the axis of the source. Results of both calculations and measurements are presented in figure 7 .

Figure 7: Comparison between measured and calculated depth dose with pinpoint

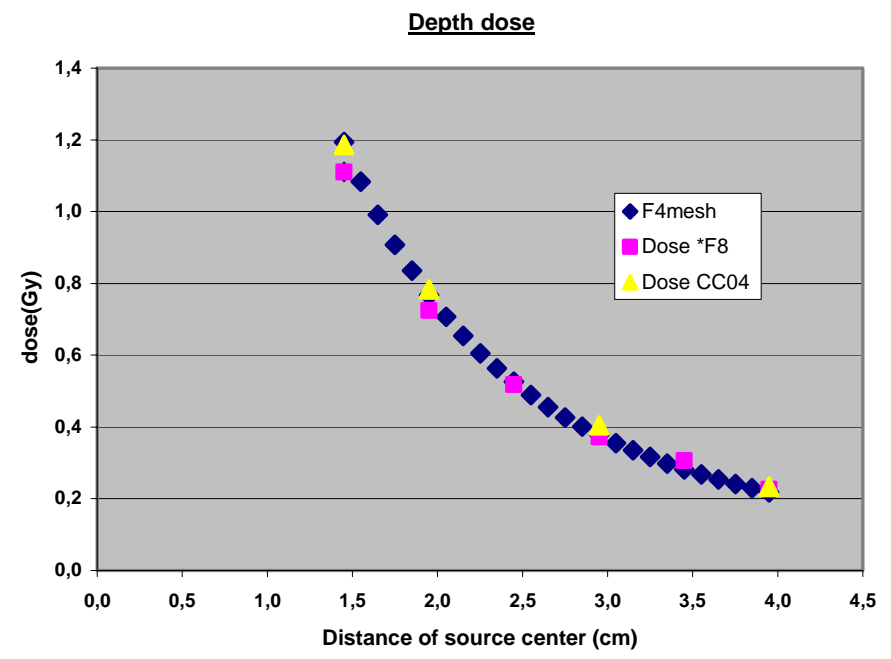


Calculated and measured results show a general good agreement. The matching seems to be better with the F4MESH calculations mostly at smaller distances; we can see differences between $0.6 \%$ for the $1.5 \mathrm{~cm}$ distance and $6 \%$ for $4 \mathrm{~cm}$ distance. Instead, comparing measurement with *F8 energy deposit tally lead to a slightly higher difference ranging from $7 \%$ at $1.5 \mathrm{~cm}$ distance to $5 \%$ at $4 \mathrm{~cm}$.

\section{Conclusions}

The PMMA sheet phantom allows a good duplication when necessary of dose measurement devices, which is of great importance to compare calculations and measurements.

Both calculated and measured isodose curves show a good agreement and also for absorbed doses. This allows us to use the MCNP5 model for dose calculations at points that are not accessible for physical measurements like on the intra-uterine tube surface.

In the future, results (measurements and calculation) will be compared with commercial treatment planning systems.

\section{Acknowledgements}

This work received financial support from the Generalitat Valenciana as well as from the Research Vice-Rector Office of the "Universidad Politécnica de Valencia" (Spain) for the stay of Isabelle Gerardy in Valencia at the "Departamento de Ingeniería Química y Nuclear" of the "Universidad Politécnica de Valencia”.

\section{REFERENCES}

[1] Nath R., Anderson L.L., Meli J.A., Olch A.J., Sitt J.A., Williamson J.F., Code of practice for brachytherapy physics: Report of the AAPM Radiation Therapy Committee Task Group No. 56, Med.Phys.24 (10), October 1997.

[2] “Java-based Nuclear Information Software (JANIS)”, http://www.nea.fr/janis/ (2005).

[3] X-5 Monte Carlo Team: MCNP - A General Monte Carlo N-Particle Transport Code, Version 5, Los Alamos National Laboratory, Los Alamos, New Mexico, USA, April 2003.

[4] NIST mass absorption coefficient table, http://physics.nist.gov/PhysRefData/XrayMassCoef/ComTab/pmma.html

[5] I. Gerardy, J. Ródenas, M. van Dycke, S. Gallardo, F. Tondeur; Application of MCNP5 code to the modelling of vaginal and intra-uterine applicators used in intracavitary brachytherapy: first approach., Journal of Physics, Conference series, vol. 102, 2008

[6] RIT113 ${ }^{\circledR}$, radiological imaging technology, 2008 